\title{
What Explains Patterns of Diversification and Richness among Animal Phyla?
}

\author{
Tereza Jezkova and John J. Wiens ${ }^{\star}$ \\ Department of Ecology and Evolutionary Biology, University of Arizona, Tucson, Arizona 85721 \\ Submitted July 6, 2016; Accepted October 13, 2016; Electronically published January 3, 2017 \\ Online enhancements: appendixes, supplemental files. Dryad data: http://dx.doi.org/10.5061/dryad.ck52b.
}

\begin{abstract}
AвSTRACт: Animal phyla vary dramatically in species richness (from one species to $>1.2$ million), but the causes of this variation remain largely unknown. Animals have also evolved striking variation in morphology and ecology, including sessile marine taxa lacking heads, eyes, limbs, and complex organs (e.g., sponges), parasitic worms (e.g., nematodes, platyhelminths), and taxa with eyes, skeletons, limbs, and complex organs that dominate terrestrial ecosystems (arthropods, chordates). Relating this remarkable variation in traits to the diversification and richness of animal phyla is a fundamental yet unresolved problem in biology. Here, we test the impacts of 18 traits (including morphology, ecology, reproduction, and development) on diversification and richness of extant animal phyla. Using phylogenetic multiple regression, the best-fitting model includes five traits that explain $\sim 74 \%$ of the variation in diversification rates (dioecy, parasitism, eyes/photoreceptors, a skeleton, nonmarine habitat). However, a model including just three (skeleton, parasitism, habitat) explains nearly as much variation ( 67\%). Diversification rates then largely explain richness patterns. Our results also identify many striking traits that have surprisingly little impact on diversification (e.g., head, limbs, and complex circulatory and digestive systems). Overall, our results reveal the key factors that shape large-scale patterns of diversification and richness across $>80 \%$ of all extant, described species.
\end{abstract}

Keywords: animals, diversification, habitat, morphology, parasitism, species richness.

\section{Introduction}

A major goal of ecology and evolutionary biology is to explain patterns of species richness. For example, why do some clades have a single species whereas others (of similar age) have more than a million? A closely related question is: what kinds of traits might be particularly important for explaining these patterns? For example, are the habitats where organisms live as important as their evolutionary innovations in morphology, development, or reproduction?

* Corresponding author; e-mail: wiensj@email.arizona.edu.

Am. Nat. 2017. Vol. 189, pp. 201-212. (C) 2017 by The University of Chicago. 0003-0147/2017/18903-57096\$15.00. All rights reserved.

DOI: $10.1086 / 690194$
Does parasitism increase the diversification rates of parasitic lineages? If so, is it as important as other traits in explaining diversity patterns?

New time-calibrated phylogenies and phylogenetic comparative methods offer exciting opportunities to address these questions. Here, we use these approaches to analyze patterns of species richness and diversification in one of the largest groups of organisms, the metazoans (i.e., animals, including $>80 \%$ of all extant described species; Roskov et al. 2016). We focus on finding the correlates of net diversification rates of clades (speciation - extinction over time) to identify traits that may be particularly important in accelerating speciation and/or buffering lineages from extinction. We focus on diversification rates rather than richness alone, since the latter ignores the ages of clades.

Animal phyla offer a compelling system in which to address these questions. First, animal phyla show dramatic variation in species richness, from a single described species in Placozoa to $>1.2$ million species in Arthropoda (Zhang 2013). Second, animals have evolved remarkable diversity in their morphology, ecology, development, and reproduction (Nielsen 2001; Hickman et al. 2012) over the past $~ 800$ million years (fig. 1). Animals range from simple, microscopic, asexual taxa found only in oceans (e.g., placozoa), to sessile, predominantly marine taxa lacking heads, eyes, limbs, and organs (e.g., sponges), to parasitic worms (e.g., many nematodes and platyhelminths), to highly mobile taxa with heads, eyes, skeletons, limbs, and complex organ systems for circulation, digestion, and excretion that now dominate terrestrial ecosystems in terms of species richness (e.g., arthropods) and body size (e.g., chordates; Nielsen 2001; Hickman et al. 2012). How this incredible variation in traits among animal phyla might be related to their striking differences in richness is a fundamental but unresolved problem in biology.

The question of which traits explain large-scale patterns of animal diversification has remained unresolved for several reasons. First, many traits have been hypothesized to play a role in explaining differences in diversification and richness among animal phyla, but their importance has not been ex- 


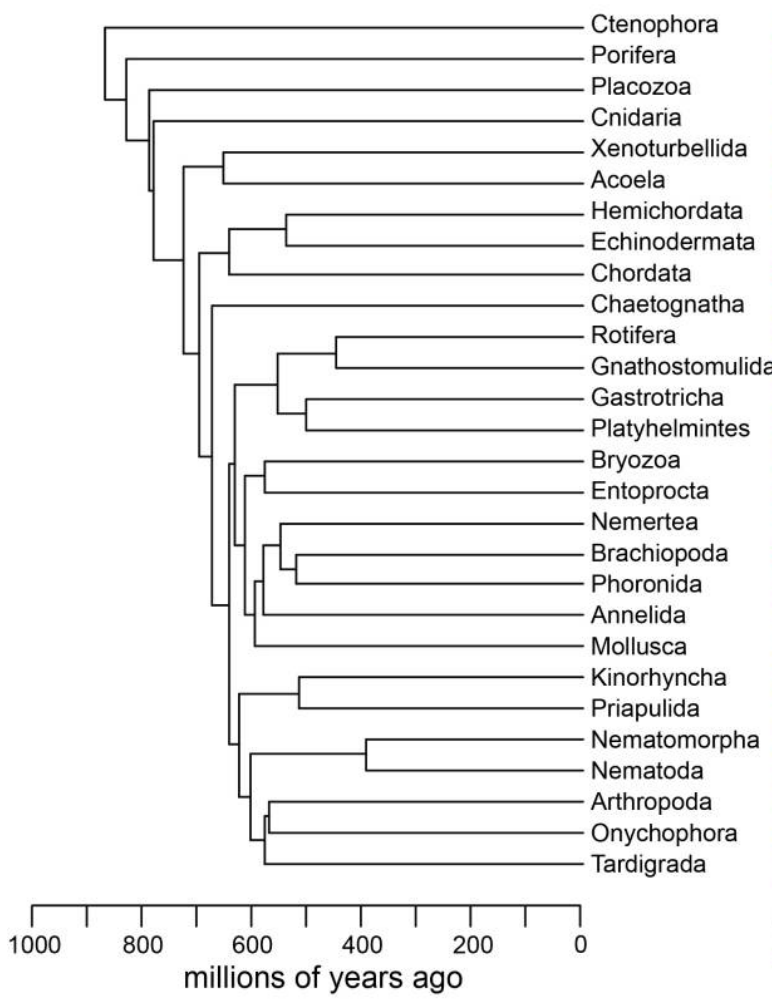

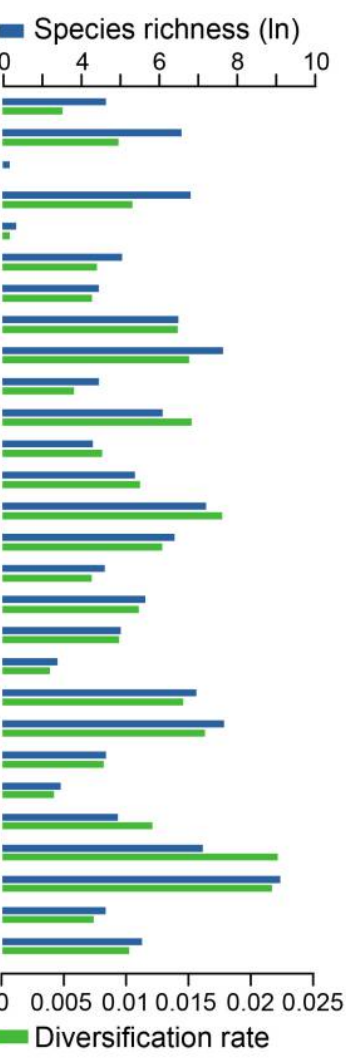

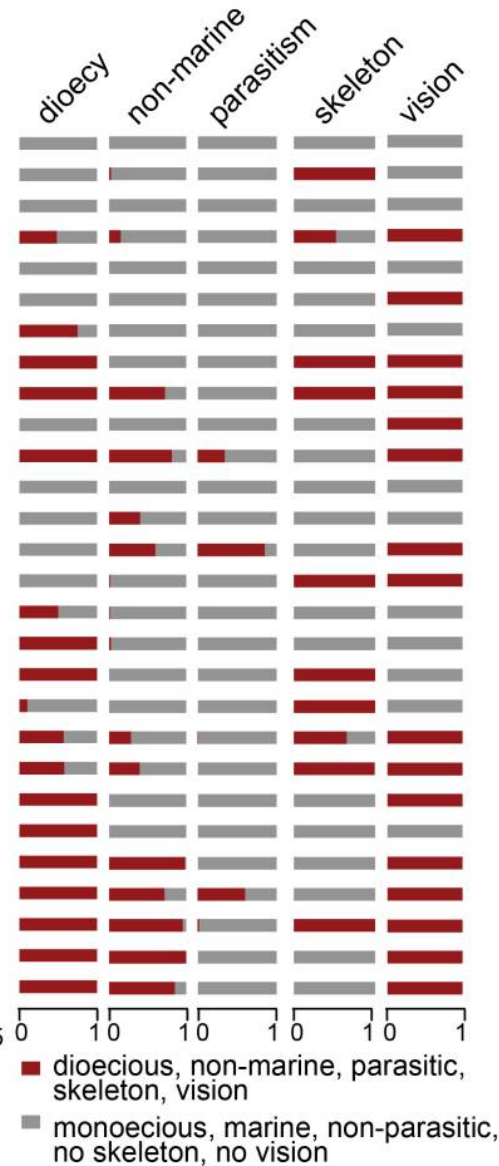

Figure 1: Summary of phylogeny, richness, diversification rates, and traits among animal phyla. Time-calibrated phylogeny of 28 animal phyla based on the topology of Dunn et al. (2014), with a root age at $\sim 836$ million years (tree 2 from Wiens 2015b). For each phylum, species richness and diversification rates (in species per million years) are shown, as well as the distributions of five traits that showed significant relationships with diversification rates: dioecy, occupancy of nonmarine habitats, parasitism, presence of a skeleton, and vision. Occupancy of terrestrial habitats is not depicted because it is largely redundant with the occupancy of nonmarine habitats (Wiens 2015b). Proportions of character states smaller than $\sim 5 \%$ are not clearly visible, but exact values are shown in supplementary file S1 (available in the Dryad Digital Repository: http://dx.doi.org/10.5061/dryad.ck52b).

plicitly tested (Heard and Hauser 1995; Mayhew 2007). Second, studies that have explicitly tested relationships between traits and diversification have usually focused on only one trait at a time (e.g., body size [Orme et al. 2002; McClain and Boyer 2009], presence of eyes [de Queiroz 1999], or occurrence in nonmarine habitats [Wiens 2015b]). Therefore, the relative importance of different traits (and the potential impact of correlations among traits) remains highly uncertain. Third, many traits that were tested showed weak or no relationship with richness or diversification (e.g., presence of eyes [de Queiroz 1999], body size [Orme et al. 2002]). Similarly, traits that were found to be significant explained only a minority of the variation in diversification rates among animal phyla (e.g., $33 \%$ for nonmarine habitat use; Wiens 2015b). Furthermore, some studies have focused on richness instead of diversification (e.g., Orme et al. 2002) without ac- counting for the fact that some clades may have more species simply because they are older. Finally, some studies have studied potentially relevant traits across animals but not at the level of phyla (e.g., de Queiroz 1999). Thus, it remains unclear how much variation in diversification rates (or richness) among phyla these traits explain.

In this article, we test the morphological, ecological, and developmental correlates of diversification among animal phyla. We utilize three time-calibrated phylogenies (Wiens $2015 b$ ) that include most (28 of 32) widely recognized phyla. We assemble a data set of 18 diverse ecological, morphological, developmental, and reproductive traits from the literature, each having the potential to impact diversification rates. We perform phylogenetic comparative analyses to identify those traits significantly related to diversification (noting that diversification rates explain $\sim 85 \%-89 \%$ of the variation in 
richness among phyla; Wiens 2015b). We then use multiple regression to identify a model explaining the maximum variation in diversification rates while including the fewest variables. We identify a set of five traits that together explain $\sim 74 \%$ of the variation in diversification rates among animal phyla and a set of three traits that explain nearly as much $(\sim 67 \%)$. Our results support the importance of ecology, a few key morphological innovations, and parasitism in explaining patterns of diversification and species richness in animals at the largest scales.

\section{Material and Methods}

\section{Traits}

We identified 18 traits that were potentially related to diversification rates of animal phyla (app. A; apps. A, B are available online). Most traits involved morphology and development, whereas a few described ecology and reproduction. These traits were previously recognized as potentially promoting diversification of animal phyla (Heard and Hauser 1995; de Queiroz 1999; Orme et al. 2002; Mayhew 2007; Wiens 2015b) or being important in animal macroevolution in general (Dunn et al. 2014). Further, we limited ourselves to those traits for which data were available from the literature for all phyla. Therefore, on the basis of this criterion, we excluded several potential traits, including diet, regenerative ability, generation time, and number of offspring. The final set included external characters (body size and symmetry; presence of eyes, legs, segmentation, and cephalization), internal characters (presence of an excretory system, circulatory system, digestive system, and an endo- or exoskeleton), characters associated with reproduction and development (asexual reproduction, dioecy, metamorphosis), and ecological characters (parasitism, occurrence in marine vs. nonmarine and terrestrial vs. aquatic habitats). Note that nonmarine includes both terrestrial and freshwater habitats, whereas aquatic includes both freshwater and marine habitats. These two characters (nonmarine and terrestrial habitat) were previously tested for relationships with diversification of animal phyla by Wiens (2015b). Similarly, Orme et al. (2002) tested for a relationship between body size and richness across animal phyla. Data for these three characters were therefore obtained primarily from Orme et al. (2002) and Wiens (2015b; but see also app. A). Data for the other 15 characters were assembled from the literature for this study.

Traits were generally scored on the basis of the estimated proportion of species in a phylum exhibiting a given state (ranging from 0 to 1 ), given that many traits varied among species within one or more phyla. In cases in which a trait showed little variation within phyla, it was treated as a categorical variable. Note that it is appropriate to perform phy- logenetic generalized least squares (PGLS) analyses with categorical independent variable as long as the dependent variable is continuous (Martins and Hansen 1997), as is the case here for diversification rates. One trait (body size) was treated as a continuous variable. Data on trait distributions among phyla were often obtained from Hickman et al. (2012), especially for the most obvious traits (e.g., head, limbs). When information was not available from Hickman et al. (2012), we searched the primary literature for additional information. Detailed information on all characters and their coding can be found in appendix A. Distributions of traits among taxa are summarized in supplementary file S1. All supplementary files are deposited in the Dryad Digital Repository: http:// dx.doi.org/10.5061/dryad.ck52b (Jezkova and Wiens 2016).

We recognize that some problems could arise when using proportions to associate traits with diversification for major clades. For example, for a given phylum with two subclades $A$ and B, a trait might be present in subclade A, but increased diversification in that phylum might be restricted to subclade B. However, such a pattern would need to be repeated within multiple phyla to give a strong relationship between that trait and diversification rates among phyla, which seems unlikely. Similarly, a trait might be present at very low frequencies within one or more phyla but could still show a relationship with diversification. Such a rare trait would be highly unlikely to be causally related to diversification across the clade. To avoid this problem, we confirmed that all traits significantly related to diversification in our results occurred in $>50 \%$ of the species in two or more phyla (fig. 1). In general, we recognize that statistical relationships between traits and diversification can support (but not prove) causation.

\section{Phylogeny and Phylum Delimitation}

We analyzed relationships between traits and diversification among the 28 animal phyla included in the three timecalibrated phylogenies of Wiens $(2015 b)$. These phyla generally correspond to the 34 phyla described by Hickman et al. (2012), with the following exceptions. The phyla Cycliophora, Loricifera, Mesozoa, and Micrognathozoa were not included, given that lack of comparable sequence data prevented their inclusion in the tree of Wiens (2015b). Acanthocephala is considered a separate phylum by Hickman et al. (2012) but is included within Rotifera here. Similarly, Sipuncula is considered a separate phylum by Hickman et al. (2012) but here was included within Annelida (for details on delimitation of phyla, see Wiens 2015b).

Time-calibrated phylogenies were used to both infer diversification rates of phyla (i.e., clade ages) and account for phylogenetic nonindependence of phyla (using phylogenetic comparative methods). We used the three time-calibrated phylogenies from Wiens (2015b). All trees used are given in 
NEXUS format in supplementary file S2. These phylogenies were based on 16 genes from 73 metazoan species, using relatively well-supported relationships among phyla as constraints. The first two trees were based on the topology of Dunn et al. (2014), which summarized animal phylogenies from many recent studies. Two sets of fossil calibration points were used, one that estimated the root of the tree at $\sim 1.3$ billion years (tree 1) and a second one that estimated the root at $836 \mathrm{Ma}$ (tree 2). For the last tree (tree 3), an alternative tree topology based largely on Philippe et al. (2011) was used, with an estimated root age of $820 \mathrm{Ma}$. Results were similar using all three trees, and we focused primarily on tree 2 (which yields a more standard set of divergence dates, along with the relatively well-established topology).

We also performed a series of secondary analyses to address the impacts of clade delimitation on the results. First, some phyla were subdivided into smaller subclades (specifically, Annelida, Arthropoda, Bryozoa, Chaetognatha, Chordata, Cnidaria, Echinodermata, Hemichordata, Mollusca, and Porifera; following Wiens 2015b), which yielded 49 higherlevel clades. We also independently tested the clades Deuterostomia (i.e., including the higher-level clades within Chordata, Echinodermata, and Hemichordata) and Ecdysozoa (including the six higher-level clades within Arthropoda and the phyla Kinorhyncha, Nematoda, Nematomorpha, Onychophora, Priapulida, and Tardigrada). However, these were not the primary analyses for our study, given that our main goal was to infer the causes of variation in diversification rates and richness among phyla (rather than simultaneously trying to explain variation both among and within phyla). For example, much of the variation within phyla might be explained by other variables not included here (i.e., that show limited variation among phyla), and traits important for explaining variation among phyla might be less important within them.

\section{Diversification Rates}

The net diversification rate for each phylum-level clade was estimated using the method of moments estimator for stem group ages (Magallón and Sanderson 2001). Estimated rates are given in supplementary file S3, along with species richness and ages of clades. The stem group estimator was used because the phylogenies included too few species for some phyla to infer their crown group age (e.g., some species-poor phyla are represented by single species). Furthermore, use of crown group ages might give a highly distorted view of a group's net diversification (e.g., a very young crown group age in an ancient group with low richness could suggest a nonsensical high diversification rate). Estimating the diversification rate of a clade using the method of moments estimator requires the clade's age, species richness, and an assumed relative extinction fraction ( $\varepsilon$, or extinction/speciation). Note that $\varepsilon$ is intended to correct for the failure to sample extinct clades entirely when extinction rates are high (Magallón and Sanderson 2001). It is not an estimate of extinction rates within extant clades. Clade ages were derived from the three time-calibrated trees. Following standard practice, we used three different values of $\varepsilon$, two extreme values ( 0 and 0.9 ), and an intermediate value (0.5). However, use of different values had relatively minor impact on the relationship between traits and diversification (supplementary file S4). Species richness estimates based on numbers of described species were obtained from Wiens (2015b). Because actual species numbers are clearly much greater than described species numbers for some phyla, we also performed a set of analyses using diversification rates estimated with projected species numbers (for details, see app. B). Specifically, we used projections of the actual number of extant species in each phylum, not merely the current number of described species.

We note that some authors have claimed that these net diversification rate estimators require that rates of diversification are constant within clades and should therefore be used only if there is a positive relationship between clade age and richness among clades (e.g., Rabosky and Adams 2012; Rabosky et al. 2012). There are two main problems with these arguments. First, the net diversification rate estimator for stem group ages is mathematically agnostic with regard to variation in diversification within clades over time. Thus, a young clade with many species will have a relatively fast net diversification rate regardless of the exact pattern of lineage accumulation over time within that clade (just as an older clade with fewer species will have a slower net rate). Second, recent simulations suggest that the accuracy of the net diversification estimator used here is similar, regardless of whether there is a positive or negative relationship between clade age and richness (Kozak and Wiens 2016). In fact, the previous studies that stated that a positive age-richness relationship was necessary (e.g., Rabosky and Adams 2012; Rabosky et al. 2012) did not directly address the accuracy of these net diversification estimators. The simulation study that did address their accuracy suggests that there can be strong relationships between true and estimated rates using these estimators and that these relationships strengthen dramatically as clade ages increase (Kozak and Wiens 2016). Importantly, the clade ages used here are much older than those simulated in that study (i.e., $\sim 15-40$ vs. $\sim 500$ million years in this study).

More generally, alternative approaches to estimating diversification rates would generally be problematic, given that the phylogenies used here include only a small fraction of the total species richness of these clades. Moreover, the approach used here allowed us to test the relationships between traits and richness and to estimate the variance in diversification rates among phyla that is explained by individual traits and combinations of traits. Such questions would be difficult to address using alternative approaches to diversification that 
do not estimate an overall diversification rate for each phylum (as done here).

\section{Testing Relationships between Traits and Diversification Rates}

The relationships between traits and diversification rates among phyla were tested using PGLS (Martins and Hansen 1997). This approach accounts for the potential statistical nonindependence of clades due to phylogeny. For PGLS, the R package CAPER (ver. 0.5.2; Orme 2013) was used. Following standard practice, the maximum likelihood transformation of branch lengths optimized for the data (lambda $=\mathrm{ML}$ ) was used, on the basis of estimated values of $\lambda$ (Pagel 1999); $\kappa$ and $\delta$ were each fixed at 1 .

We first tested the relationships between diversification rates and each of the 18 traits separately. The analysis was repeated using the three phylum-level topologies and the three values of $\varepsilon$. Results were largely insensitive to different topologies and $\varepsilon$ values.

We then conducted multiple regression analyses, including only traits that showed a significant relationship with diversification rates of phyla when tested individually for tree 2 (which has a relatively standard topology and divergence dates) and $\varepsilon=0.5$ (the intermediate value). For the multiple regression analyses, we first included all traits that were significantly related to diversification in separate analyses in a single multiple regression model. We then repeated the analysis, after excluding one trait at a time (until all $n-1$ combinations were reached, where $n$ is the number of traits). From these analyses, we selected the analysis with the lowest Akaike Information Criterion (AIC) value. We then excluded one additional trait at a time from this analysis until all possible combinations were tested. We then again selected the analysis with the lowest AIC. We repeated these steps until only a single pair of traits remained. From these analyses, we then selected the model with the lowest AIC value overall, which should simultaneously maximize the variance explained and minimize the number of variables included. We also used the $P$ values associated with each individual trait in the multiple regression analyses to assess whether each variable significantly contributed to the best-fitting analysis.

We recognize that other approaches to model selection are possible. For example, we could have initially included all traits, regardless of their relationship to diversification in pairwise analyses, and sequentially removed them. However, this would create an extremely large number of models to compare (i.e., all the possible combinations of traits from those including 17 traits down to those with only two), with most combinations almost certainly being unhelpful (since most variables show no significant relationship with diversification). Adding other traits might increase the $r^{2}$ slightly, but such models would also be penalized for their extra parameters.
The relationships between individual traits and diversification rates were further explored with three additional analyses. First, we repeated the analyses of individual traits for 28 phyla using projected species richness values instead of numbers of described species (for details, see app. B and supplementary file S5). Second, we repeated the analyses of individual traits using the 49 higher-level clades, after subdividing some phyla into subclades (for details, see Wiens [2015b] and supplementary file S6). These additional analyses were conducted using tree 2 and all three values of $\varepsilon$. Third, we repeated the analyses individually for Annelida, Arthropoda, and Mollusca (i.e., analyzing variation among the higher-level clades within these phyla) and for all subdivided clades within Ecdysozoa (12 clades total) and Deuterostomia (seven clades). We specifically tested those characters that showed significant relationships with diversification rate in the main analysis of 28 phyla. This latter set of analyses was conducted using tree 2 and the intermediate value of $\varepsilon$. Results are described in supplementary file S7.

\section{Results}

We first separately tested each of 18 traits (table 1 ; supplementary file S4) for their potential impacts on diversification. Only six traits showed significant relationships with diversification rates across different trees and diversification analyses (table 1; supplementary file S4). The distribution of these traits across the phylogeny is summarized in figure 1, along with patterns of diversification rates and species richness among phyla (note that nonmarine and terrestrial habitat use are redundant and not shown separately; see below). The presence of photoreceptors and/or eyes in a phylum (vision hereafter) explained $36 \%-39 \%(P<.001-.001)$ of the variation in diversification rates (ranges summarize results across the three trees and three different $\varepsilon$ values used for estimating diversification rates for each tree). An alternative coding for vision, using the proportion of species with eyes only (i.e., excluding photoreceptors), was also significantly related to diversification $\left(r^{2}=0.23-0.32, P=.002-.010\right)$, but this coding explained less variation than when eyes and photoreceptors were combined into a single state. Phyla with higher proportions of nonmarine species (i.e., freshwater and terrestrial) and higher proportions of terrestrial species had significantly higher diversification rates. Occurrence in nonmarine and terrestrial habitats explained 30\%-37\% $(P=.001-.002)$ and $25 \%-28 \%(P=.003-.006)$, respectively, of the variation in diversification rates, as previously reported (Wiens $2015 b$ ). Diversification rates were also significantly related to the presence of dioecy $\left(r^{2}=0.15-0.22, P=.006-.046\right)$, a skeleton (either internal or external; $r^{2}=0.15-0.26, P=$ $.012-.050)$, and the proportion of parasitic species in a clade $\left(r^{2}=0.16-0.27, P=.005-.038\right)$. 
Table 1: Results of phylogenetic generalized least squares analyses individually testing relationships between 18 traits and diversification rates of animal phyla

\begin{tabular}{lll}
\hline Trait & $r^{2}$ & $P$ \\
\hline Proportion of species exhibiting sexual reproduction & .03 & .354 \\
Presence or absence of cephalization & .06 & .209 \\
Presence or absence of a circulatory system & .04 & .323 \\
Presence or absence of coelom & .01 & .672 \\
Presence of a digestive system with two openings & .09 & .111 \\
Proportion of dioecious species &. $\mathbf{1 7}$ & $\mathbf{. 0 2 7}$ \\
Presence or absence of an excretory organ & .12 & .072 \\
Presence or absence of legs & .08 & .153 \\
Size: median biovolume (mm ${ }^{3}$; ln transformed) & .02 & .516 \\
Proportion of species undergoing metamorphosis & .04 & .300 \\
Proportion of nonmarine species &. $\mathbf{3 7}$ & $\mathbf{. 0 0 1}$ \\
Proportion of parasitic species &. $\mathbf{2 6}$ & $\mathbf{. 0 0 5}$ \\
Presence or absence of segmentation & .09 & .123 \\
Proportion of species that have a skeleton &. $\mathbf{1 4}$ & $\mathbf{. 0 4 7}$ \\
Presence or absence of bilateral symmetry & .06 & .214 \\
Proportion of terrestrial species &. $\mathbf{2 7}$ & $\mathbf{. 0 0 5}$ \\
Proportion of vagile species & .01 & .597 \\
Presence or absence of photoreceptors/eyes &. $\mathbf{3 8}$ & $<.001$ \\
\hline
\end{tabular}

Note: Brief descriptions of each trait and regression results are shown. The results are for tree 2 and the intermediate relative extinction fraction $(\varepsilon=0.5)$. Results for other trees and $\varepsilon$ values are in supplementary file S4 (available in the Dryad Digital Repository: http://dx.doi.org/10.5061 /dryad.ck52b). Correlation coefficients $\left(r^{2}\right)$ and $P$ values are shown, with significant values in bold.

Most other traits were not significantly related to diversification across any trees or $\varepsilon$ values (supplementary file S4). However, the presence of a digestive tract with two openings was significantly related to higher diversification rates on one tree (tree 3; see "Material and Methods") when using lower $\varepsilon$ values $(0,0.5)$.

We then conducted multiple regression analyses (table 2; supplementary file S8) to identify the combination of traits that best explained patterns of diversification among phyla. For simplicity, we conducted these analyses only on the preferred tree (tree 2) and an intermediate $\varepsilon$ value (0.5), given that different trees and $\varepsilon$ values gave similar results in pairwise analyses (supplementary file S4). We included five of the six traits that were each significantly related to diversification rates (table 1; supplementary file S4). We excluded terrestrial habitat because it is largely redundant with nonmarine habitat (Wiens 2015b). We compared models with different combinations of traits using the AIC.

The most variation in diversification rates (74\%) was explained (and the lowest AIC obtained [152.5]) when all five traits were included (vision, parasitism, skeleton, dioecy, nonmarine habitat; table 2). However, separate analyses that individually excluded vision, dioecy, nonmarine habitat, or dioecy and vision each resulted in only a small decrease in explained variation and in similar model fit. In particular, $71 \%$ of the variation was explained when either vision $(\mathrm{AIC}=153.8)$, dioecy $($ AIC $=153.5)$, or nonmarine habitat $($ AIC $=153.3)$ was excluded (table 2). Importantly, $67 \%$ of the variation was still explained when both dioecy and vision were excluded (table 2), with a negligible increase in AIC (154.9). In comparison, excluding parasitism or the skeleton resulted in a model with substantially worse fit and less variation explained (with parasitism excluded: AIC $=164.1, r^{2}=0.58$; with skeleton excluded: AIC $\left.=163.0, r^{2}=0.59\right)$. Thus, a model including only nonmarine habitat, skeleton, and parasitism seemed to maximize fit and explanatory power with the fewest traits.

In the analysis that included all five traits, only parasitism $(P=.001)$ and the presence of a skeleton $(P=.002)$ significantly contributed to the model, whereas vision $(P=.11)$, dioecy $(P=.13)$, and nonmarine habitat $(P=.14)$ did not (table 2). This pattern presumably occurred because nonmarine habitat is significantly related to both vision and dioecy (vision and nonmarine: $r^{2}=0.33, P=.001$; dioecy and nonmarine: $r^{2}=0.21, P=.014$; for all pairwise relationships among these five traits, see supplementary file S9). These relationships between nonmarine habitat, vision, and dioecy also explain why $r^{2}$ and AIC did not improve substantially when each one of these three traits was removed (table 2).

We also performed additional analyses to further explore our main results. First, we used projected species numbers (supplementary file S5) to estimate the diversification rate for each phylum instead of numbers of described species 
Table 2: Results of phylogenetic multiple regression analyses of relationships between diversification rates of animal phyla and selected traits

\begin{tabular}{|c|c|c|c|}
\hline Traits included in model & $r^{2}$ & AIC & $P$ \\
\hline Dioecy, nonmarine habitat, parasitism, skeleton, vision & .740 & 152.50 & $<.001$ \\
\hline Dioecy & & & .130 \\
\hline Nonmarine habitat & & & .144 \\
\hline Parasitism & & & .001 \\
\hline Skeleton & & & .002 \\
\hline Vision & & & .114 \\
\hline Nonmarine habitat, parasitism, skeleton, vision & .710 & 153.48 & $<.001$ \\
\hline Nonmarine habitat & & & .028 \\
\hline Parasitism & & & .003 \\
\hline Skeleton & & & .002 \\
\hline Vision & & & .097 \\
\hline Dioecy, parasitism, skeleton, vision & .712 & 153.28 & $<.001$ \\
\hline Dioecy & & & .025 \\
\hline Parasitism & & & .000 \\
\hline Skeleton & & & .003 \\
\hline Vision & & & .019 \\
\hline Dioecy, nonmarine habitat, parasitism, skeleton & .708 & 153.75 & $<.001$ \\
\hline Dioecy & & & .111 \\
\hline Nonmarine habitat & & & .024 \\
\hline Parasitism & & & .001 \\
\hline Skeleton & & & .001 \\
\hline Nonmarine habitat, parasitism, skeleton & .673 & 154.91 & $<.001$ \\
\hline Nonmarine habitat & & & .001 \\
\hline Parasitism & & & .002 \\
\hline Skeleton & & & .001 \\
\hline
\end{tabular}

Note: The five models with the best fit on the basis of Akaike Information Criterion (AIC) values are shown (for full results, see supplementary file S8, available in the Dryad Digital Repository: http://dx.doi.org/10.5061/dryad.ck52b). The results are based on tree 2 and an $\varepsilon$ of 0.5 . Correlation coefficients $\left(r^{2}\right)$, AIC, and $P$ values are shown for each model, along with $P$ values for each trait in each model.

(utilizing tree 2 and all three $\varepsilon$ values). This analysis (supplementary file S5) supported relationships between diversification and five of the six traits supported in the analyses based on described species (vision: $r^{2}=0.32-0.33, P=$ .001-.002; parasitism: $r^{2}=0.35, P=.001$; dioecy: $r^{2}=$ $0.15-0.17, P=.031-.046$; nonmarine habitat: $r^{2}=0.36-$ $0.37, P=.001$; terrestrial habitat: $r^{2}=0.26-0.27, P=$ .004-.006). However, the presence of a skeleton was nonsignificant across all three $\varepsilon$ values. Parasitism explained more variation when projected species richness was used than using described richness, presumably because of the high projected richness of parasitic nematodes (supplementary file S5). The remaining five significant variables explained equal or less variation when projected species richness was used, relative to diversification rates estimated from numbers of described species.

Second, we subdivided many of the more diverse phyla to yield a total of 49 clades (Wiens 2015b). Using tree 2 and three $\varepsilon$ values, we found that three traits still showed significant relationships with diversification rates (vision: $r^{2}=$ 0.21-0.23, $P<.001$; nonmarine habitat: $r^{2}=0.11-0.17$, $P=.001-.006$; terrestrial habitat: $r^{2}=0.15-0.21, P=$
.001-.006; supplementary file S6). The relationship between diversification rates and all three traits is weaker for 49 clades in comparison with 28 clades, as expected given the problem of simultaneously explaining diversification rates both within and between phyla (see above).

Finally, we tested for relationships between traits and diversification rates separately within selected clades (supplementary file S7). Specifically, we tested the six candidate traits within the phyla Annelida ( $n=5$ clades), Arthropoda $(n=6)$, and Mollusca $(n=5)$ and within the clades Deuterostomia ( $n=7$ clades, including the subdivided clades of Chordata, Echinodermata, and Hemichordata) and Ecdysozoa $(n=12$ clades, including the subdivided clades of Arthropoda and the phyla Kinorhyncha, Nematoda, Nematomorpha, Onychophora, Priapulida, and Tardigrada). Although many traits that were important among phyla were not significantly related to diversification rates within these clades, we found a significant relationship with the presence of a skeleton within Annelida $\left(r^{2}=0.83, P=.03\right)$. We also found a significant relationship between diversification rates and nonmarine habitat within Mollusca $\left(r^{2}=0.91, P=.01\right.$; see also Wiens $2015 b)$. However, sample sizes of clades were very small 
within these groups, and some characters were largely invariant within them.

\section{Discussion}

In this study, we analyzed the morphological, developmental, and ecological traits that potentially underlie patterns of diversification and species richness among clades in the dominant group of living organisms (animals). Specifically, we evaluated 18 traits for their potential to explain variation in diversification rates among animal phyla. We identified five traits that together explain $\sim 74 \%$ of the variation in diversification rates among animal phyla (table 2). These traits include morphological innovations (skeleton, eyes/photoreceptors), ecological characteristics (occurrence in nonmarine habitats, parasitism), and a trait associated with reproduction (dioecy). However, a model including just three traits (habitat, parasitism, skeleton) explained nearly as much variation ( 67\%). To our knowledge, this may be the first study to show the importance of parasitism to patterns of diversification at the largest phylogenetic scales. Perhaps just as importantly, we identified many striking traits that have surprisingly little impact on the diversification rates of animal phyla. These included heads, limbs, body size, vagility, sexuality (vs. asexuality), metamorphosis, and complex organ systems used for circulation, digestion, and excretion (table 1). Overall, our results show the importance of both ecology and morphological innovations in explaining large-scale patterns of diversity and diversification across $>80 \%$ of all described species.

Our results also demonstrate the importance of considering multiple traits when explaining diversity patterns. First, by including multiple variables, we were able to explain most $(74 \%)$ of the variation in diversification rates among animal phyla, with a relatively limited number of variables (five). Each variable separately explained only a limited amount of variation. Further, most of this variation could be explained by three variables alone. In contrast, previous studies of diversification among animal phyla that considered only a single trait (nonmarine habitat) were able to explain only $30 \%-37 \%$ of the variation in diversification rates among anima phyla (Wiens 2015b). Importantly, our results also suggest that an analysis of a single trait might conclude that a trait is important, when in fact the trait that is more directly influencing diversification is one that is correlated with it. In our analysis, there were five traits with seemingly strong effects on diversification, but three appeared to be overlapping in their effects (dioecy, vision, nonmarine habitat). Thus, multiple regression analyses that excluded one of these three traits had only slightly higher AIC scores and explained only slightly less variation relative to a model including all five (table 2). Pairwise comparisons showed these traits to be significantly related in their distributions among phyla (vision and nonmarine: $r^{2}=0.33, P=.001$; dioecy and nonmarine: $r^{2}=0.21, P=.014$; supplementary file S9). Indeed, vision is believed to have facilitated terrestrial invasion, and dioecy is sometimes considered an adaptation to terrestrial life (Little 1983). The former idea is supported by our post hoc ancestral state reconstructions of vision and habitat on the tree, which suggest that vision evolved before the invasion of nonmarine habitats (supplementary file S10). In contrast, parasitism and the presence of a skeleton seem to be independent of both each other and these other traits, given the weak relationships among them (supplementary file S9). An important goal for future studies should be to tease out the relative effects of these traits on patterns of animal diversification, possibly by analyzing patterns within the more species-rich phyla that vary for these traits (e.g., annelids, chordates, molluscs, arthropods). For example, Wiens (2015b) found that nonmarine habitats explained $>90 \%$ of the variation in diversification rates among major clades within molluscs. We found this same pattern and that the other traits analyzed were not significantly related to large-scale diversification patterns in molluscs. Similarly, we found that the presence of a skeleton explained $83 \%$ of the variation in diversification rates among major clades of annelids, whereas the other traits analyzed did not (supplementary file S7).

We acknowledge that other traits might be important in explaining richness and diversification patterns beyond the ones that we included here. Furthermore, the effects of these other traits might be masked by their correlations with traits that we included. Nevertheless, we included a large number of dramatic traits that varied among phyla (e.g., heads, limbs, major organ systems) and showed that most were unrelated to diversification. Furthermore, the traits that we did include explained most variation in diversification rates among phyla. This suggests that other traits - if they are unrelated to those we included - would have little additional variation left to explain. However, explaining richness patterns within phyla will likely require including additional traits or might show different traits to be important relative to those that explained diversification rates among phyla. For example, among major vertebrate clades, terrestrial habitat use explains the majority of variation in diversification rates $(\sim 66 \%)$, whereas nonmarine habitat use has no significant impact (Wiens 2015a). Similarly, our analyses of 49 subdivided clades and within select phyla supported only some of the traits that were found to be important across animal phyla.

We recognize that some readers may be dismayed that our study is not based on fossil taxa. However, our goal here was to explain patterns of net diversification and extant species richness among animal phyla. Thus, even if patterns of species richness and diversification in fossil taxa were different from those analyzed here (e.g., Alroy 2010), those are not the patterns that we are trying to explain. Furthermore, our primary focus is on explaining extant species richness across 
phyla, not the diversity of every subclade of every phylum at every slice of time over the past $\sim 800$ million years. Of course, analyses within phyla and analyses utilizing fossils may be critically important in further testing (and disentangling) the importance of these factors. We merely assert that our primary question was related to the richness, net diversification, and traits of living taxa. Furthermore, many of the traits analyzed here would be difficult to incorporate using fossil information (e.g., dioecy, parasitism). Similarly, species richness would also be difficult to estimate for many phyla, given the bias of the fossil record toward hard-bodied organisms (Foote et al. 2007).

Our study raises many questions for future research. We think that the most important is, how exactly does each of these traits increase diversification? There are several relevant hypotheses in the literature, depending on the trait. First, nonmarine environments may offer more effective barriers to dispersal, which may promote speciation (May 1994; Benton 2001; Vermeij and Grosberg 2010; CarreteVega and Wiens 2012; Wiens 2015b). Other hypotheses to explain lower marine richness (and diversification) have also been proposed (May 1994; Benton 2001; Vermeij and Grosberg 2010) but are more ambiguous, given available data, such as higher terrestrial productivity or the difficulty of moving in water (Carrete-Vega and Wiens 2012; Wiens 2015b). Marine extinction may also be important (CarreteVega and Wiens 2012; Wiens 2015b). Vision may facilitate entering new adaptive zones, such as nonmarine habitat, and may increase the ability of organisms to localize prey, predators, and conspecific mates (de Queiroz 1999). Dioecy may facilitate internal fertilization and therefore transitions to terrestrial environments, where external fertilization is problematic (Little 1983). Further, dioecy may increase the evolutionary potential of species by increasing their levels of heterozygosity and genetic diversity through genetic recombination (Lloyd 1980). The evolution of the skeleton has been traditionally associated with the Cambrian explosion because it might have provided body support and defense from predators (Bengtson and Zhao 1992). Consequently, the presence of a skeleton is believed to have promoted rapid diversification of many animal phyla during the Cambrian (Thomas et al. 2000; Erwin et al. 2011). There has been some discussion of the idea that parasitism and similar types of species interactions might promote diversification (e.g., Yoder and Nuismer 2010; Althoff et al. 2014; Hembry et al. 2014), but strong empirical support at broad scales has been elusive (e.g., Wiegmann et al. 1993). To our knowledge, ours is the first study to support this hypothesis at such a deep phylogenetic scale. One potential mechanism by which parasitism might promote diversification is through cospeciation of hosts and their parasites (either strict or relatively loose), especially if niche partitioning within a host enables coexistence of multiple parasite species in a single host (e.g., Feder and Forbes
2010). Another potential mechanism is host switching (e.g., Ricklefs et al. 2004, 2014). We also note that our definition of parasitism was quite restrictive (i.e., an obligatory relationship between two heterospecific organisms during which the parasite is metabolically dependent on the host; app. A). More inclusive definitions might show an even greater impact of parasitism on diversification (e.g., encompassing herbivorous insects; Ehrlich and Raven 1964; Mitter et al. 1988; Futuyma and Agrawal 2009; Wiens et al. 2015). This process also raises a methodological issue, in that increased diversification in phylogenetically distant host and parasite lineages might be coupled rather than independent. For example, the high overall diversification rate of nematodes might be causally related to the high diversification rate of the arthropods that many nematodes parasitize (especially when using our projected richness values), rather than there being fully independent increases in each clade. Finally, another interesting topic for future research is how these traits might interact with each other and with large-scale historical factors, such as mass extinction events, climatic changes, and the rise of angiosperms.

Our results support the conclusions of some previous studies on higher-level animal diversification but not others. Our results agree with those of Wiens (2015b) on the importance of habitat (but with the caveat that the effects of habitat, dioecy, and vision are difficult to distinguish). Our results also agree with those of Orme et al. (2002) in showing that body size is not important. Orme et al. (2002) found no significant relationship between their index of body size for animal phyla (median biovolume among species) and the species richness of these phyla. In contrast, McClain and Boyer (2009) found a significant relationship between extreme body sizes of species within phyla (minimum and maximum among species) and the species richness of phyla. However, if body sizes evolved randomly across a tree, one would expect clades with more species to have both larger and smaller species (as a result of chance alone), even if there were no causal relationship between size and diversification. Therefore, we did not perform the same tests as McClain and Boyer (2009) to avoid this potential artifact. Our results disagree with those of de Queiroz (1999), who found no significant relationship between the presence of eyes and diversification. Our results support significant relationships between diversification rate and vision (but again with the important caveat that the effects of vision and habitat are difficult to parse). De Queiroz (1999) did not analyze phyla but compared pairs of lower-level sister clades. He speculated that eyes might have been important at the level of phyla (as in our study) but not more recently within phyla. Also, de Queiroz (1999) assessed only the impact of image-forming eyes on diversification, whereas we included both eyes and photoreceptors. We found that including both eyes and photoreceptors revealed a stronger relationship with diversification. 
In conclusion, we found that a limited number of traits can explain most variation in diversification rates among animal phyla. These traits include morphological innovations (i.e., skeleton) as well as ecology (i.e., nonmarine habitat). Our results also support parasitism as a key process promoting large-scale patterns of animal diversification. This latter result further supports the idea that local-scale species interactions help drive large-scale patterns of clade diversification over hundreds of million years (e.g., Wiens et al. 2015). We also show that numerous dramatic evolutionary innovations failed to drive diversification patterns among animal phyla (including the evolution of a head, limbs, motility, sexuality, and complex organs systems for circulation, digestion, and excretion). Future research should address the specific processes by which the traits supported here increase diversification, research that will presumably occur at smaller phylogenetic scales. Importantly, our results demonstrate which traits do (and do not) scale up to explain these large-scale diversity patterns, regardless of patterns at smaller scales.

\section{Acknowledgments}

T.J. was supported by a Postdoctoral Excellence in Research and Teaching fellowship (5K12GM000708-13). We thank Y. Michalakis, S. B. Heard, and two anonymous reviewers for many helpful comments that improved the manuscript.

\section{Literature Cited}

Alroy, J. 2010. The shifting balance of diversity among major marine animal groups. Science 329:1191-1194.

Althoff, D. M., K. A. Segraves, and M. T. J. Johnson. 2014. Testing for coevolutionary diversification: linking pattern with process. Trends in Ecology and Evolution 29:82-89.

Bengtson, S., and Y. Zhao. 1992. Predatorial borings in late Precambrian mineralized exoskeletons. Science 257:367-369.

Benton, M. J. 2001. Biodiversity on land and in the sea. Geological Journal 36:211-230.

Carrete-Vega, G., and J. J. Wiens. 2012. Why are there so few fish in the sea? Proceedings of the Roval Society B 279:2323-2329.

de Queiroz, A. 1999. Do image-forming eyes promote evolutionary diversification? Evolution 53:1654-1664.

Dunn, C. W., G. Giribet, G. D. Edgecombe, and A. Hejnol. 2014. Animal phylogeny and its evolutionary implications. Annual Review of Ecology, Evolution, and Systematics 45:371-395.

Ehrlich, P. R., and P. H. Raven. 1964. Butterflies and plants: a study in coevolution. Evolution 18:586-608.

Erwin, D. H., M. Laflamme, S. M. Tweedt, E. A. Sperling, D. Pisani, and K. J. Peterson. 2011. The Cambrian conundrum: early divergence and later ecological success in the early history of animals. Science 334 : 1091-1097.

Feder, J. L., and A. A. Forbes. 2010. Sequential speciation and the diversity of parasitic insects. Ecological Entomology 35:67-76.

Foote, M., A. I. Miller, D. M. Raup, and S. M. Stanley. 2007. Principles of paleontology. Macmillan, New York
Futuyma, D. J., and A. A. Agrawal. 2009. Macroevolution and the biological diversity of plants and herbivores. Proceedings of the National Academy of Sciences of the USA 106:18054-18061.

Heard, S. B., and D. L. Hauser. 1995. Key evolutionary innovations and their ecological mechanisms. Historical Biology 10:151-173.

Hembry, D. H., J. B. Yoder, and K. R. Goodman. 2014. Coevolution and the diversification of life. American Naturalist 184:425-438.

Hickman, C., L. Roberts, S. Keen, A. Larson, and D. Elsenhour. 2012. Animal diversity. 6th ed. McGraw-Hill, New York.

Jezkova, T., and J. J. Wiens. 2016. Data from: What explains patterns of diversification and richness among animal phyla? Dryad Digital Repository, http://dx.doi:10.5061/dryad.ck52b.

Kozak, K. H., and J. J. Wiens. 2016. Testing the relationships between diversification, species richness, and trait evolution. Systematic Biology 65:975-988.

Little, C. 1983. The colonisation of land: origins and adaptations of terrestrial animals. Cambridge University Press, Cambridge.

Lloyd, D. G. 1980. Benefits and handicaps of sexual reproduction. Evolutionary Biology 13:69-111.

Magallón, S., and M. J. Sanderson. 2001. Absolute diversification rates in angiosperm clades. Evolution 55:1762-1780.

Martins, E. P., and T. F. Hansen. 1997. Phylogenies and the comparative method: a general approach to incorporating phylogenetic information into the analysis of interspecific data. American Naturalist 149:646-667.

May, R. M. 1994. Biological diversity: differences between land and sea. Philosophical Transactions of the Royal Society B 343:105-111.

Mayhew, P. J. 2007. Why are there so many insect species? perspectives from fossils and phylogenies. Biological Reviews of the Cambridge Philosophical Society 82:425-454.

McClain, C. R., and A. G. Boyer. 2009. Biodiversity and body size are linked across metazoans. Proceedings of the Roval Society B 276: 2209-2215.

Mitter, C., B. Farrell, and B. Wiegmann. 1988. The phylogenetic study of adaptive zones: has phytophagy promoted insect diversification? American Naturalist 132:107-128.

Nielsen, C. 2001. Animal evolution: interrelationships of living phyla Oxford University Press, Oxford.

Orme, C. D. L., D. L. J. Quicke, J. M. Cook, and A. Purvis. 2002. Body size does not predict species richness among the metazoan phyla. Journal of Evolutionary Biology 15:235-247.

Orme, D. 2013. The caper package: comparative analysis of phylogenetics and evolution in R. R package version 0.5.2.

Pagel, M. 1999. Inferring the historical patterns of biological evolution. Nature 401:877-884.

Philippe, H., H. Brinkmann, D. V. Lavrov, D. T. J. Littlewood, M. Manuel, G. Woerheide, and D. Baurain. 2011. Resolving difficult phylogenetic questions: why more sequences are not enough. PLoS Biology 9:e1000602.

Rabosky, D. L., and D. C. Adams. 2012. Rates of morphological evolution are correlated with species richness in salamanders. Evolution 66:1807-1818.

Rabosky, D. L., G. J. Slater, and M. E. Alfaro. 2012. Clade age and species richness are decoupled across the eukaryotic tree of life. PLoS Biology 10:e1001381.

Ricklefs, R. E., S. M. Fallon, and E. Bermingham. 2004. Evolutionary relationships, cospeciation, and host switching in avian malaria parasites. Systematic Biology 53:111-119.

Ricklefs, R. E., D. C. Outlaw, M. Svensson-Coelho, M. C. I. Medeiros, V. A. Ellis, and S. Latta. 2014. Species formation by host shifting 
in avian malaria parasites. Proceedings of the National Academy of Sciences of the USA 111:14816-14821.

Roskov, Y., L. Abucay, T. Orrell, D. Nicolson, C. Flann, N. Bailly, P. Kirk, et al. 2016. Species 2000 and ITIS Catalogue of Life: 27th February 2016. Species 2000: Naturalis, Leiden. www.catalogueoflife.org/col.

Thomas, R. D. K., R. M. Shearman, and C. W. Stewart. 2000. Evolutionary exploitation of design options by the first animals with hard skeletons. Science 288:1239-1242.

Vermeij, G. J., and R. K. Grosberg. 2010. The great divergence: when did diversity on land exceed that in the sea? Integrative and Comparative Biology 50:675-682.

Wiegmann, B. M., C. Mitter, and B. Farrell. 1993. Diversification of carnivorous parasitic insects: extraordinary radiation or specialized dead end? American Naturalist 142:737-754.

Wiens, J. J. 2015a. Explaining large-scale patterns of vertebrate diversity. Biology Letters 11:20150506.

2015b. Faster diversification on land than sea helps explain global biodiversity patterns among habitats and animal phyla. Ecology Letters 18:1234-1241.

Wiens, J. J., R. T. Lapoint, and N. K. Whiteman. 2015. Herbivory increases diversification across insect clades. Nature Communications 6:8370.

Yoder, J. B., and S. L. Nuismer. 2010. When does coevolution promote diversification? American Naturalist 176:802-817.

Zhang, Z.-Q. 2013. Animal biodiversity: an update of classification and diversity in 2013. Zootaxa 3703:5-11.

\section{References Cited Only in the Online Appendixes and Supplements}

Ali, M. A., ed. 1984. Photoreception and vision in invertebrates. Plenum, New York.

Appeltans, W., S. T. Ahyong, G. Anderson, M. V. Angel, T. Artois, N. Bailly, R. Bamber, et al. 2012. The magnitude of global marine species diversity. Current Biology 22:2189-2202.

Autrum, H., M. F. Bennet, B. Diehn, K. Hamdorf, M. Heisenberg, M. Järviletho, P. Kunze, et al. 1979. Comparative physiology and evolution of vision in invertebrates. A. Invertebrate photoreceptors. Springer, Berlin.

Basset, Y., L. Cizek, P. Cuénoud, R. K. Didham, F. Guilhaumon, O. Missa, V. Novotny, et al. 2012. Arthropod diversity in a tropical forest. Science 338:1481-1484.

Bertolani, R. 2001. Evolution of the reproductive mechanisms in tardigrades: a review. Zoologischer Anzeiger 240:247-252.

Boyle, P. R. 1969. Fine structure of the eyes of Onithochiton neglectus (Mollusca: Polyplacophora). Zeitschrift für Zellforschung und Mikroskopische Anatomie 102:313-332.

Burnham, K. P., and D. R. Anderson. 2004. Multimodel inference: understanding AIC and BIC in model selection. Sociological Methods and Research 33:261-304.

Burr, A. H. 1984. Evolution of eyes and photoreceptor organelles in lower phyla. Pages 131-178 in M. A. Ali, ed. Photoreception and vision in invertebrates. Plenum, New York.

Camino, N., and M. Achinelly. 2011. Biodiversity of insect-parasitic nematodes in soil pest insect (Orthoptera, Gryllidae and Gryllotalpidae) in wheat fields of Buenos Aires, Argentina. Anales de Biología 33:15-21.

Chapman, A. D. 2009. Numbers of living species in Australia and the world. 2nd ed. Australian Biodiversity Information Services, Toowoomba.
Cheng, T. C. $1986 a$. Acanthocephala: the spiny-headed worms. Pages 445-464 in T. C. Cheng, ed. General parasitology. 2nd ed. Academic Press, San Diego, CA.

. 1986b. Cestoidea: the tapeworms Cestodaria: the unsegmented tapeworms. Pages 378-386 in T. C. Cheng, ed. General parasitology. 2nd ed. Academic Press, San Diego, CA.

. 1986c. Eucestoda: the true tapeworms. Pages 387-444 in T. C. Cheng, ed. General parasitology. 2nd ed. Academic Press, San Diego, CA.

- 1986d. Introduction to the parasitic arthropods. Pages 572591 in T. C. Cheng, ed. General parasitology. 2nd ed. Academic Press, San Diego, CA.

1986e. The mites. Pages 592-613 in T. C. Cheng, ed. General parasitology. 2nd ed. Academic Press, San Diego, CA. . 1986f. Other zooparasites. Pages 706-787 in T. C. Cheng, ed. General parasitology. 2nd ed. Academic Press, San Diego, CA. 1986g. Parasitism and symbiosis. Pages 1-34 in T. C. Cheng, ed. General parasitology. 2nd ed. Academic Press, San Diego, CA. Coe, W. R. 1930. Asexual reproduction in nemerteans. Physiological Zoology 3:297-308.

Deland, C., C. B. Cameron, K. P. Rao, W. E. Ritter, and T. H. Bullock. 2010. A taxonomic revision of the family Harrimaniidae (Hemichordata: Enteropneusta) with descriptions of seven species from the Eastern Pacific. Zootaxa 2408:1-30.

Edmondson, C. H. 1935. Autotomy and regeneration in Hawaiian starfishes. Occasional Papers of the Bishop Museum Honolulu 11:1-29.

Erwin, T. L. 1982. Tropical forests their richness in Coleoptera and other arthropod species. Coleopterists Bulletin 36:74-75.

Fournier, A. 1984. Photoreceptors and photosensitivity in Platyhelminthes. Pages 217-239 in M. A. Ali, ed. Photoreception and vision in invertebrates. Plenum, New York.

Gilbert, L., ed. 1981. Metamorphosis: a problem in developmental biology. 2nd ed. Plenum, New York.

Goto, T., and M. Yoshida. 1984. Photoreception in Chaetognatha. Pages 727-742 in M. A. Ali, ed. Photoreception and vision in invertebrates. Plenum, New York.

Greven, H. 2007. Comments on the eyes of tardigrades. Arthropod Structure and Development 36:401-407.

Grucmanová, Š., and J. Holuša. 2013. Nematodes associated with bark beetles, with focus on the genus Ips (Coleoptera: Scolytinae) in Central Europe. Acta Zoologica Bulgarica 65:547-556.

Hinde, R. T. 1998. The Cnidaria and Ctenophora. Pages 28-57 in D. T. Anderson, ed. Invertebrate zoology. Oxford University Press, Oxford.

Lambert, K., and S. Bekal. 2002. Introduction to plant-parasitic nematodes. Plant Health Instructor, doi:10.1094/PHI-I-2002-1218-01.

Lambshead, P. J. D., and G. Boucher. 2003. Marine nematode deep-sea biodiversity: hyperdiverse or hype? Journal of Biogeography 30:475485.

Lammert, V. 1985. The fine structure of protonephridia in Gnathostomulida and their comparison within Bilateria. Zoomorphology 105: 308-316.

Maddison, W. P., and D. R. Maddison. 2011. Mesquite: a modular system for evolutionary analysis. Version 2.75 . http://mesquiteproject.org.

Maslakova, S. A. 2010. Development to metamorphosis of the nemertean pilidium larva. Frontiers in Zoology 7:1-18.

Menzel, R. 1979. Spectral sensitivity and color vision in invertebrates. Pages 504-580 in H. Autrum, ed. Comparative physiology and evolution of vision in invertebrates. A. Invertebrate photoreceptors. Springer, Berlin. 


\section{The American Naturalist}

Nakano, H., K. Lundin, S. J. Bourlat, M. J. Telford, P. Funch, J. R. Nyengaard, M. Obst, et al. 2013. Xenoturbella bocki exhibits direct development with similarities to Acoelomorpha. Nature Communications 4:1-6.

Nielsen, C., and K. Worsaae. 2010. Structure and occurrence of Cyphonautes larvae (Bryozoa, Ectoprocta). Lournal of Morphology 271: 1094-1109.

Novotny, V., Y. Basset, S. E. Miller, G. D. Weiblen, B. Bremer, L. Cizek, and P. Drozd. 2002. Low host specificity of herbivorous insects in a tropical forest. Nature 416:841-844.

Poinar, G. O., Jr. 1977. A synopsis of the nematodes occurring in blackflies (Diptera: Simuliidae). Bulletin of the World Health Organization 55:509-515.

2012. Nematode parasites and associates of ants: past and present. Psyche 2012:192017.

Poulin, R., and S. Morand. 2004. Parasite biodiversity. Smithsonian Institution, Washington, DC.

Powers, T. O., D. A. Neher, P. Mullin, A. Esquivel, R. M. Giblin-Davis, N. Kanzaki, S. P. Stock, et al. 2009. Tropical nematode diversity: vertical stratification of nematode communities in a Costa Rican humid lowland rainforest. Molecular Ecology 18:985-996.

Purschke, G. 2005. Sense organs in polychaetes (Annelida). Pages 5378 in T. Bartolomaeus and G. Purschke, eds. Morphology, molecules, evolution and phylogeny in Polychaeta and related taxa. Springer, Berlin.

Ruppert, E. E., R. S. Fox, and R. D. Barnes. 2004. Invertebrate zoology: a functional evolutionary approach. 7th ed. Thomson, Brooks/Cole New York.

Saito-Morooka, F. 2014. The prevalence of the parasitic nematode Sphaerularia sp. in the overwintering gynes of Parapolybia spp. (Hymenoptera, Polistinae). Journal of Hvmenoptera Research 38: $37-43$.
Shigeno, S., T. Sasaki, T. Moritaki, T. Kasugai, M. Vecchione, and K. Agata. 2008. Evolution of the cephalopod head complex by assembly of multiple molluscan body parts: evidence from Nautilus embryonic development. Journal of Morphology 269:1-17.

Stork, N. E., J. McBroom, C. Gely, and A. J. Hamilton. 2015. New approaches narrow global species estimates for beetles, insects, and terrestrial arthropods. Proceedings of the National Academv of Sciences of the USA 112:7519-7523.

Tautz, D. 2004. Segmentation. Developmental Cell 7:301-312.

Thomsen, E., and E. Hakansson. 1995. Sexual versus asexual dispersal in clonal animals: examples from cheilostome bryozoans. Paleobiology 21:496-508.

Wade, C. M., P. B. Mordan, and B. Clarke. 2001. A phylogeny of the land snails (Gastropoda: Pulmonata). Proceedings of the Roval Society B 268:413-422.

Wald, G. 1981. Metamorphosis: an overview. Pages 1-39 in L. Gilbert, ed. Metamorphosis: a problem in developmental biology. Plenum, New York

Wallace, R. L. 1980. Ecology of sessile rotifers. Hydrobiologia 73:181193.

Walter, D. E., and H. C. Proctor. 2013. Mites: ecology, evolution, and behaviour. Life at a microscale. 2 nd ed. Springer, Berlin.

Webb, M. 1969. An evolutionary concept of some sessile and tubicolous animals. Sarsia 38:1-8.

WoRMS Editorial Board. 2015. World register of marine species http://www.marinespecies.org. Accessed December 1, 2015.

Yoshida, M. 1979. Extraocular photoreception. Pages 582-650 in H. Autrum, ed. Comparative physiology and evolution of vision in invertebrates. A. Invertebrate photoreceptors. Springer, Berlin.

Associate Editor: Stephen B. Heard Editor: Yannis Michalakis

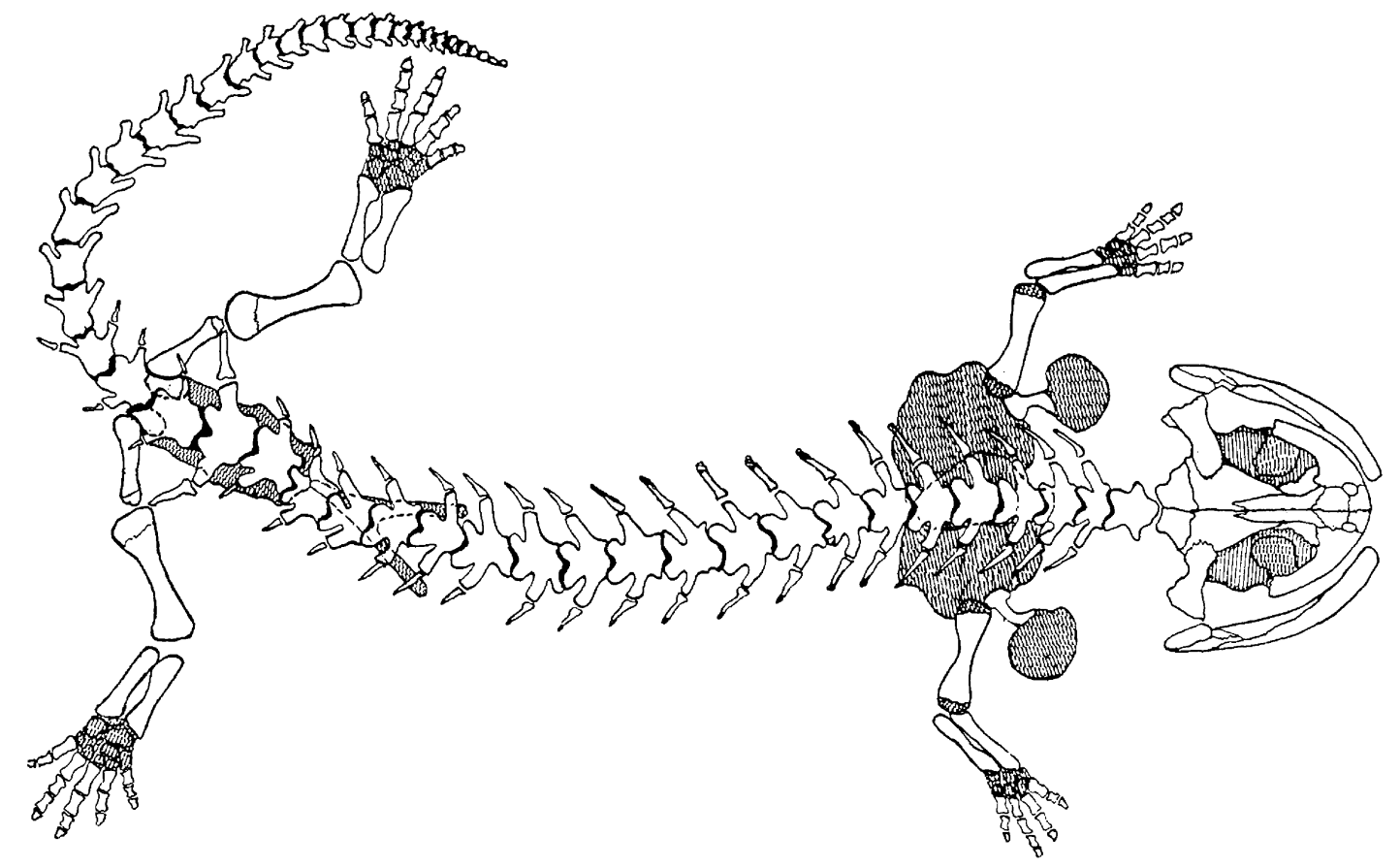

"In the skull of the modern Caudata we find an arrangement of the elements which is quite similar to that described for the Branchiosauria. In the skull of Megalobatrachus [shown], for instance, we find the following bones paired in the median line: the premaxillæ, the nasals, the frontals, the parietals, and on the posterior end of the skull occur the exoccipitals." From "The Ancestry of the Caudate Amphibia" by Roy L. Moodie (The American Naturalist 1908, 42:361-373). 